\title{
Three-dimensional smoothed-particle hydrodynamics simulation of deformation characteristics in slope failure
}

\author{
Y. AN*, Q. WU*, C. SHI* and Q. LIU*
}

\begin{abstract}
This paper presents the development, validation and application of a smoothed-particle hydrodynamics model for three-dimensional (3D) simulation of the evolution of large deformation failure in geomaterials. The Drucker-Prager model with non-associated plastic flow rules is implemented into the smoothed-particle hydrodynamics formulations to describe elasto-plastic soil behaviour. Two typical numerical examples - a two-dimensional (2D) analysis of cohesive slope instability and a 3D simulation for instant collapse of a granular slope - are shown to demonstrate the effectiveness of the method for modelling large deformation of slope failure. Good agreement with experimental observations and previous simulated results is obtained in terms of the profile and internal deformation, respectively. The method is then applied to two special 3D slopes with different geometric configurations, including a curving slope surface and a slope that turns corners. The results suggest that $3 \mathrm{D}$ effects should be considered for natural landslides. By influencing the stress status, slope geometries have a significant effect on the final profile, slip surface and distance. The results provide a more accurate and detailed reference for landslide evaluation and foundation ditch design.
\end{abstract}

KEYWORDS: failure; numerical modelling; slopes

\section{INTRODUCTION}

Landslides accompanied by large deformation and flow-like failure of geo-materials are common disasters which often result in serious loss of life and property. Analysis based on numerical simulation is one of the powerful research techniques for providing information regarding: $(a)$ when and where the failure will take place; $(b)$ what the slip surface will look like; and $(c)$ how far the collapsed soil mass will flow. Although numerical analysis has been proven to be successful with respect to $(a)$ and $(b)$, the third question regarding the post-failure stage is still open and attracts much attention. Mesh-based numerical methods, such as the finite-difference method (FDM) and the finite-element method (FEM), dominate the simulation of steady and quasi-steady problems at the present time. For example, after the pioneering work of Griffiths \& Marquez (2007) on three-dimensional (3D) slope stability analysis using FEM, Nian et al. (2012) and Zhang et al. (2013), using the FDM and FEM methods, respectively, discussed the effects of geometries on 3D slope stability. However, these methods often suffer from serious numerical difficulties in dealing with slope post-failure problems, which are accompanied by extremely large deformations, free surfaces and crack extensions. Severe mesh winding, twisting and distortion may lead to non-physical results, even the interruption of calculation. The discrete-element method (DEM) can overcome these deficiencies and solve large deformation problems (Tapias et al., 2015). However, the friction and elastic parameters of the contact zone in DEM need to be calibrated as they are not directly obtained from experiments. The coupled Eulerian-Lagrangian (CEL) method can handle multiphase and large deformation problems (Dey et al., 2015), but for

Manuscript received 9 October 2015; revised manuscript accepted 16 March 2016.

Discussion on this paper is welcomed by the editor.

* Key Laboratory for Mechanics in Fluid Solid Coupling Systems, Institute of Mechanics, Chinese Academy of Sciences, Beijing, People's Republic of China. this method the mesh must be drawn in the whole computation area, as in the Euler method, which results a huge mesh in 3D simulation. Thus, novel numerical methods which can overcome these numerical difficulties are necessary to simulate the overall processes of slope failure.

To overcome the numerical difficulties, mesh-free methods based on continuum mechanics have been developed to investigate large deformations in slope failure evolution. Mesh-free methods characterise materials by a series of random distributions of particles and rebuild the particle interactions when particles move. Instead of solving the discrete governing equations on predefined grids, mesh-free methods solve the discrete equations on dynamic particle connections, and therefore have obvious advantages in dealing with large deformation and free surfaces (see Liu \& Liu, 2004). Smoothed-particle hydrodynamics (SPH) is one of the most famous methods because of its relatively mature development and wide applications. Two typical techniques are usually adopted in the SPH framework to simulate the large deformation of geo-materials. The first is generally developed for modelling flow-like soil behaviours (Pastor et al., 2009; Huang et al., 2012; Cascini et al., 2014; Hu et al., 2015). The Navier-Stokes equation with Bingham rheology model is used as the governing equation, and the material parameters such as strength are attributed to the rheology model and its parameters. Despite the success of this method in both two-dimensional (2D) and 3D run-out analysis of flow-like landslides, it cannot reasonably simulate slip surface development and crack propagation owing to its fluid nature. The second technique is developed from geotechnical plastic mechanics (Bui et al., 2008, 2011; Bui \& Fukagawa, 2013; Chen \& Qiu, 2014). As an elasto-plastic constitutive model is implemented into SPH formulations, this method can handle more general soil mechanics problems, especially the entire slope failure evolution; that is, from stable slope to instability, to collapse, flow and finally back to a stable state. Furthermore, it can also capture the slip surface shape and crack extension relatively well. However, most of the current studies are confined to the 2D case, and few of them apply this method to the dynamic analysis of $3 \mathrm{D}$ slope failure, 
owing to its complex numerical implementation and huge consumption of computational effort.

Natural slope failure always evolves in three dimensions. It is therefore important to build a 3D model for both small and large deformation of geo-materials and to analyse the differences between the $2 \mathrm{D}$ and $3 \mathrm{D}$ cases in terms of the final profile, slip surface and distance. This can provide a more reasonable reference for evaluating landslide disasters and economically designing infrastructures, for example, excavations and embankments. The motivation of this paper is to establish a SPH model for both 2D and 3D simulation of large deformation failure evolution in geo-materials. Bui et al. (2008) undertook the pioneering work in which the elasto-plastic constitutive model for the soils was introduced into the SPH framework and 2D simulation of large granular material deformation was achieved. Based on their work, the present authors have developed this method for a 3D case. The Drucker-Prager model with non-associated plastic flow rules is implemented into the SPH formulations to describe the elasto-plastic soil behaviour. One difficulty in extending this model from two dimensions to three dimensions is in designing proper artificial terms to dampen the numerical instability. In this paper, the unified expression for 2D and $3 \mathrm{D}$ artificial stress is implemented and related parameters are calibrated. In the following sections, the development of the model is first presented, followed by model validation and two case studies.

\section{NUMERICAL IMPLEMENTATION}

Based on the original work of Bui et al. (2008), their method is extended and applied to the 3D analysis of slope post-failure features. Assuming isotropic soil, the Drucker-Prager model with non-associated plastic flow rules is adopted to describe the complex soil elasto-plastic behaviour. The 3D SPH model for simulating large deformation after slope failure is described in detail as follows, including governing equations, the constitutive model, SPH formulation and explicit time integration.

\section{Governing equations}

The governing equations of geo-materials consist of mass and momentum conservation

$$
\left\{\begin{array}{l}
\frac{D \rho}{D t}=-\rho \frac{\partial v^{\alpha}}{\partial x^{\alpha}} \\
\frac{D v^{\alpha}}{D t}=\frac{1}{\rho} \frac{\partial \boldsymbol{\sigma}^{\alpha \beta}}{\partial x^{\beta}}+f^{\alpha}
\end{array}\right.
$$

where $\alpha, \beta$ denote the Cartesian components $x, y, z$ with the Einstein convention applied to repeated indices; $\rho$ is soil density; $v$ is soil velocity; $f^{\alpha}$ is the component of acceleration caused by external force, which is the gravitational acceleration in this work; $\boldsymbol{\sigma}$ represents the total stress tensor of soil and its expression can be obtained from the constitutive model.

\section{Constitutive model}

In geotechnical mechanics, the total strain rate tensor is defined as

$$
\dot{\boldsymbol{\varepsilon}}^{\alpha \beta}=\frac{1}{2}\left(\frac{\partial v^{\alpha}}{\partial x^{\beta}}+\frac{\partial v^{\beta}}{\partial x^{\alpha}}\right)
$$

Moreover, the total strain rate tensor can be decomposed into an elastic and a plastic part

$$
\dot{\boldsymbol{\varepsilon}}^{\alpha \beta}=\dot{\boldsymbol{\varepsilon}}_{\mathrm{e}}^{\alpha \beta}+\dot{\boldsymbol{\varepsilon}}_{\mathrm{p}}^{\alpha \beta}
$$

The elastic strain rate tensor $\dot{\boldsymbol{\varepsilon}}_{\mathrm{e}}^{\alpha \beta}$ is normally computed by the generalised Hooke's law

$$
\dot{\boldsymbol{\varepsilon}}_{\mathrm{e}}^{\alpha \beta}=\frac{\dot{S}^{\alpha \beta}}{2 G}+\frac{1-2 v}{3 E} \dot{\boldsymbol{\sigma}}^{\gamma \gamma} \delta^{\alpha \beta}
$$

where $\dot{\boldsymbol{\sigma}}^{\gamma \gamma}$ is the sum of three principal stress rates

$$
\dot{\boldsymbol{\sigma}}^{y y}=\dot{\boldsymbol{\sigma}}^{x x}+\dot{\boldsymbol{\sigma}}^{y y}+\dot{\boldsymbol{\sigma}}^{z z}
$$

Similarly, $\dot{\boldsymbol{\varepsilon}}^{\eta \gamma}$ is the sum of three principal strain rates

$$
\dot{\boldsymbol{\varepsilon}}^{y y}=\dot{\boldsymbol{\varepsilon}}^{x x}+\dot{\boldsymbol{\varepsilon}}^{y y}+\dot{\boldsymbol{\varepsilon}}^{z z}
$$

By applying the plastic flow rule, the plastic strain rate $\dot{\boldsymbol{\varepsilon}}_{\mathrm{p}}^{\alpha \beta}$ is defined as

$$
\dot{\boldsymbol{\varepsilon}}_{\mathrm{p}}^{\alpha \beta}=\dot{\lambda} \frac{\partial g}{\partial \boldsymbol{\sigma}^{\alpha \beta}}
$$

The soil hydrostatic pressure $P$ can be obtained directly from the constitutive equation by the standard definition of mean stress

$$
P=-\frac{\boldsymbol{\sigma}^{\gamma y}}{3}=-\frac{1}{3}\left(\boldsymbol{\sigma}^{x x}+\boldsymbol{\sigma}^{y y}+\boldsymbol{\sigma}^{z z}\right)
$$

The Drucker-Prager model with non-associated plastic flow rules is adopted here. The yield condition $f\left(I_{1}, J_{2}\right)$ and plastic potential function $g\left(I_{1}, J_{2}\right)$ have the following forms, respectively

$$
\begin{aligned}
& f\left(I_{1}, J_{2}\right)=\sqrt{J_{2}}+\alpha_{\phi} I_{1}-k_{\mathrm{c}}=0 \\
& g\left(I_{1}, J_{2}\right)=\sqrt{J_{2}}+\alpha_{\psi} I_{1}-C
\end{aligned}
$$

where $I_{1}$ and $J_{2}$ are, respectively, the first and second invariants of the stress tensor; $C$ is an arbitrary constant; $\alpha_{\phi}$ and $k_{\mathrm{c}}$ are Drucker-Prager's constants, which are related to the Coulomb's material constants $c$ (cohesion) and $\phi$ (internal friction); $\alpha_{\psi}$ has the same expression as $\alpha_{\phi}$ and is related to the dilatancy angle $\psi$ of geo-materials.

$$
\begin{gathered}
\alpha_{\phi}=\frac{\tan \phi}{\sqrt{9+12 \tan ^{2} \phi}} k_{\mathrm{c}}=\frac{3 c}{\sqrt{9+12 \tan ^{2} \phi}} \\
\alpha_{\psi}=\frac{\tan \psi}{\sqrt{9+12 \tan ^{2} \psi}}
\end{gathered}
$$

for the 2D plane strain condition.

$$
\begin{gathered}
\alpha_{\phi}=\frac{2 \sin \phi}{\sqrt{3}(3-\sin \phi)} k_{\mathrm{c}}=\frac{6 c \cos \phi}{\sqrt{3}(3-\sin \phi)} \\
\alpha_{\psi}=\frac{2 \sin \psi}{\sqrt{3}(3-\sin \psi)}
\end{gathered}
$$

for the $3 \mathrm{D}$ condition in which the Drucker-Prager failure surface coincides with the outer cone of the Mohr-Coulomb hexagonal surface, corresponding to triaxial compression. Comparing with plastic deformation, smaller elastic deformation of volume can be ignored in the present model. The plastic deformation of volume can be represented by the dilatancy angle, $\psi$.

The stress-strain relationship is therefore expressed as

$$
\begin{aligned}
\frac{D \boldsymbol{\sigma}_{i}^{\alpha \beta}}{D t}= & 2 G \dot{\boldsymbol{e}}_{i}^{\alpha \beta}+K \boldsymbol{\varepsilon}_{i}^{\gamma \gamma} \delta_{i}^{\alpha \beta} \\
& -\dot{\lambda}_{i}\left(3 \alpha_{\psi} K \delta^{\alpha \beta}+\frac{G}{\sqrt{J_{2}}} \boldsymbol{s}_{i}^{\alpha \beta}\right)
\end{aligned}
$$

where $\dot{\lambda}$ is the rate of change of the plastic multiplier and is determined by the elasto-plastic function, equation (9); 
$\dot{\boldsymbol{s}}^{\alpha \beta}$ is the deviatoric shear stress rate tensor; $\dot{\boldsymbol{e}}_{i}^{\alpha \beta}$ is the deviatoric shear strain rate tensor; $\delta$ is Kronecker's delta; $K$ and $G$ are, respectively, the elastic bulk modulus and the shear modulus, which are related to the Young's modulus, $E$, and Poisson ratio, $v$, through the following equations

$$
K=\frac{E}{3(1-2 v)} \text { and } G=\frac{E}{2(1+v)}
$$

A general technique to treat finite strain problems is to compute a field variable for the next time step by way of its rate of change during the current time step. Considering the effect of rigid body rotation on the current stress rate, the Jaumann rate of Cauchy stress is used here

$$
\dot{\boldsymbol{\sigma}}_{J}^{\alpha \beta}=\dot{\boldsymbol{\sigma}}^{\alpha \beta}-\dot{\boldsymbol{\omega}}^{\alpha \gamma} \boldsymbol{\sigma}^{\gamma \beta}-\dot{\boldsymbol{\omega}}^{\beta \gamma} \boldsymbol{\sigma}^{\alpha \gamma}
$$

where ' $'$ ' denotes the derivative with respect to time; subscript $J$ designates the Jaumann rate; and $\dot{\boldsymbol{\omega}}$ is the spin rate tensor

$$
\dot{\boldsymbol{\omega}}^{\alpha \beta}=\frac{1}{2}\left(\frac{\partial v^{\alpha}}{\partial x^{\beta}}-\frac{\partial v^{\beta}}{\partial x^{\alpha}}\right)
$$

The stress rate tensor in a finite strain framework is converted to

$$
\dot{\boldsymbol{\sigma}}^{\alpha \beta}=\dot{\boldsymbol{\sigma}}_{J}^{\alpha \beta}+\dot{\boldsymbol{\omega}}^{\alpha \gamma} \boldsymbol{\sigma}^{\gamma \beta}+\dot{\boldsymbol{\omega}}^{\beta \gamma} \boldsymbol{\sigma}^{\alpha \gamma}
$$

The integration of rate equations is dependent on the assumption of infinitesimal strain between two adjacent configurations. This formulation is adopted in the current study and this treatment is acceptable as long as the time increments are small.

Finally, the normal form of the stress-strain relationship for elasto-plastic materials can be expressed as

$$
\begin{aligned}
\frac{D \boldsymbol{\sigma}^{\alpha \beta}}{D t}= & \boldsymbol{\sigma}^{\alpha \gamma} \dot{\boldsymbol{\omega}}^{\beta \gamma}+\boldsymbol{\sigma}^{\gamma \beta} \dot{\boldsymbol{\omega}}^{\alpha \gamma}+2 G \dot{\boldsymbol{e}}^{\alpha \beta}+K \boldsymbol{\varepsilon}^{\gamma \gamma} \delta^{\alpha \beta} \\
& -\dot{\lambda}\left(3 \alpha_{\psi} K \delta^{\alpha \beta}+\frac{G}{\sqrt{J_{2}}} \boldsymbol{s}^{\alpha \beta}\right)
\end{aligned}
$$

where the two first terms are the results from the Jaumann stress rate tensor, the third and fourth terms refer to the elastic behaviour, and the last term relates to the plastic deformation.

\section{SPH formulation}

In the SPH simulation, the entire calculation domain is expressed by finite but sufficient discretised particles with physical properties such as volume, density, velocity, acceleration and stress. The interaction between particles is realised by a kernel function. Through kernel and particle approximations, the governing equations and the constitutive equation become a set of ordinary differential equations that only depend on the variable of time. Then, the other field variables can be obtained by explicit time integration. The discretised equations in SPH formulation can be expressed as follows.

The continuity equation

$$
\frac{D \rho_{i}}{D t}=\sum_{j=1}^{N} m_{j}\left(v_{i}^{\alpha}-v_{j}^{\alpha}\right) \frac{\partial W_{i j}}{\partial x_{i}^{\alpha}}
$$

The momentum equation

$$
\begin{aligned}
\frac{D v_{i}^{\alpha}}{D t}= & \sum_{j=1}^{N} m_{j}\left(\frac{\boldsymbol{\sigma}_{i}^{\alpha \beta}+\boldsymbol{\sigma}_{j}^{\alpha \beta}}{\rho_{i} \rho_{j}}-\prod_{i j} \delta^{\alpha \beta}+F_{i j}^{n} \boldsymbol{R}_{i j}^{\alpha \beta}\right) \\
& \times \frac{\partial W_{i j}}{\partial x_{i}^{\beta}}+g^{\alpha}
\end{aligned}
$$

The constitutive equation

$$
\begin{aligned}
\frac{D \boldsymbol{\sigma}_{i}^{\alpha \beta}}{D t}= & \boldsymbol{\sigma}_{i}^{\alpha \gamma} \dot{\boldsymbol{\omega}}^{\beta \gamma}+\boldsymbol{\sigma}_{i}^{\gamma \beta} \dot{\boldsymbol{\omega}}_{i}^{\alpha \gamma}+2 G \dot{\boldsymbol{e}}_{i}^{\alpha \beta}+K \boldsymbol{\varepsilon}_{i}^{\gamma \gamma} \delta_{i}^{\alpha \beta} \\
& -\dot{\lambda}_{i}\left(3 \alpha_{\psi} K \delta^{\alpha \beta}+\frac{G}{\sqrt{J_{2}}} \boldsymbol{s}_{i}^{\alpha \beta}\right)
\end{aligned}
$$

The rate of change of the plastic multiplier

$$
\dot{\lambda}_{i}= \begin{cases}\frac{3 \alpha_{\phi} K \dot{\boldsymbol{\varepsilon}}_{i}^{\gamma \gamma}+\left(G / \sqrt{\boldsymbol{J}_{2}}\right) \mathbf{s}_{i}^{\alpha \beta} \dot{\boldsymbol{\varepsilon}}_{i}^{\alpha \beta}}{9 \alpha_{\phi} \alpha_{\psi} K+G} & f\left(I_{1}, J_{2}\right)>0 \\ 0 & f\left(I_{1}, J_{2}\right) \leq 0\end{cases}
$$

The strain rate tensor and the spin rate tensor

$$
\begin{aligned}
& \dot{\boldsymbol{\varepsilon}}_{i}^{\alpha \beta}=\frac{1}{2}\left[\sum_{j=1}^{N} \frac{m_{j}}{\rho_{j}}\left(v_{j}^{\alpha}-v_{i}^{\alpha}\right) \frac{\partial W_{i j}}{\partial x_{i}^{\beta}}+\sum_{j=1}^{N} \frac{m_{j}}{\rho_{j}}\left(v_{j}^{\beta}-v_{i}^{\beta}\right) \frac{\partial W_{i j}}{\partial x_{i}^{\alpha}}\right] \\
& \dot{\boldsymbol{\omega}}_{i}^{\alpha \beta}=\frac{1}{2}\left[\sum_{j=1}^{N} \frac{m_{j}}{\rho_{j}}\left(v_{j}^{\alpha}-v_{i}^{\alpha}\right) \frac{\partial W_{i j}}{\partial x_{i}^{\beta}}-\sum_{j=1}^{N} \frac{m_{j}}{\rho_{j}}\left(v_{j}^{\beta}-v_{i}^{\beta}\right) \frac{\partial W_{i j}}{\partial x_{i}^{\alpha}}\right]
\end{aligned}
$$

$W_{i j}$ is the kernel function. To suppress the tension instability in the calculation, the kernel function proposed by Yang \& Liu (2012) is used in this paper. Its second derivative is non-negative.

$$
\begin{aligned}
W & =(r, h) \\
& =\alpha_{\mathrm{D}}\left\{\begin{array}{ll}
q^{3}-6 q+6, & 0 \leq q<1 \\
(2-q)^{3}, & 1 \leq q<2 \\
0, & q \geq 2
\end{array} \quad q=\frac{r}{h}\right.
\end{aligned}
$$

where the normalised coefficient $\alpha_{\mathrm{D}}$ is $1 /\left(3 \pi h^{2}\right)$ in the $2 \mathrm{D}$ condition and $15 /\left(62 \pi h^{3}\right)$ in the $3 \mathrm{D}$ case.

The artificial viscosity term $\Pi$ is used to stabilise the numerical system. It is given by

$$
\prod_{i j}= \begin{cases}\frac{-\alpha \bar{c}_{i j} \mu_{i j}+\beta \mu_{i j}^{2}}{\bar{\rho}_{i j}} & v_{i j} \cdot x_{i j}<0 \\ 0 & v_{i j} \cdot x_{i j} \geq 0\end{cases}
$$

where $\mu_{i j}=\left(h_{i j} \cdot v_{i j} \cdot r_{i j}\right) /\left(r_{i j}^{2}+0 \cdot 01 h_{i j}^{2}\right) ; \quad \bar{\rho}_{i j}=\left(\rho_{i}+\rho_{j}\right) / 2$; $\bar{c}_{i j}=\left(c_{\mathrm{s} i}+c_{\mathrm{s} j}\right) / 2 ; \quad h_{i j}=\left(h_{i}+h_{j}\right) / 2 ; \quad v_{i j}=v_{i}-v_{j} ; \quad r_{i j}=r_{i}-r_{j} ;$ $c_{\mathrm{s}}=\sqrt{4 G / 3 \rho+K / \rho} ; \alpha$ and $\beta$ are constants and contingent upon specific problems. The artificial viscosity term would cause too much energy dissipation if used with an $\alpha$ value that is too large. Alternatively, the term would not be enough to suppress the numerical instability if used with an $\alpha$ value that is too small. For the trial method, the authors chose $\alpha=0 \cdot 1$ and $\beta=0$ in this study.

The artificial stress term $F_{i j}^{n} \boldsymbol{R}_{i j}^{\alpha \beta}$ in equation (20) is used to reduce tensile instability, where $F_{i j}=W_{i j} / W(\Delta \boldsymbol{x}, h)$; $n=W(0, h) / W(\Delta \boldsymbol{x}, h), n=2.55$ in this paper; $\boldsymbol{R}_{i j}^{\alpha \beta}=\boldsymbol{R}_{i}^{\alpha \beta}+\boldsymbol{R}_{j}^{\alpha \beta}$, where $\boldsymbol{R}_{i}^{\alpha \beta}$ and $\boldsymbol{R}_{j}^{\alpha \beta}$ are, respectively, the components of the artificial stress tensor at particles $i$ and $j$. A unified expression for $2 \mathrm{D}$ and $3 \mathrm{D}$ artificial stress is derived here. Assuming $\boldsymbol{\sigma} \boldsymbol{x}=\eta \boldsymbol{x}, \eta$ is invariant, and $\boldsymbol{x}$ is a non-zero $m$-dimensional vector, then $\eta_{1}, \eta_{2}, \ldots, \eta_{m}$ are eigenvalues corresponding to vector $\boldsymbol{x} . \boldsymbol{\sigma}^{\prime}=\operatorname{diag}\left(\eta_{1}, \eta_{2}, \ldots, \eta_{m}\right), m=2$ for the $2 \mathrm{D}$ condition, and $m=3$ for the $3 \mathrm{D}$ case.

$$
\boldsymbol{R}^{\alpha \beta}= \begin{cases}-\varepsilon \frac{\boldsymbol{\sigma}^{\prime \alpha \beta}}{\rho^{2}} & \boldsymbol{\sigma}^{\prime \alpha \beta}>0 \\ 0 & \boldsymbol{\sigma}^{\prime \alpha \beta} \leq 0\end{cases}
$$


where $\varepsilon$ is a constant taken from 0 to 1 ; here the constant is taken to be $\varepsilon=0 \cdot 3$ after careful calibration.

\section{Explicit time integration}

The Verlet scheme is adopted in the proposed model. To discretise equations, this time-stepping algorithm is split into two parts. In general, variables are calculated according to

$$
\begin{aligned}
& v_{n+1}=v_{n-1}+2 \Delta t\left(\frac{D v}{D t}\right)_{n} ; \rho_{n+1}=\rho_{n-1}+2 \Delta t\left(\frac{D \rho}{D t}\right)_{n} \\
& \boldsymbol{\sigma}_{n+1}=\sigma_{n-1}+2 \Delta t\left(\frac{D \boldsymbol{\sigma}}{D t}\right)_{n} ; r_{n+1}=r_{n}+\Delta t \cdot v_{n}+0 \cdot 5 \Delta t^{2}\left(\frac{D v}{D t}\right)_{n}
\end{aligned}
$$

Once every $M$ time steps $(M=40$, here $)$, variables are calculated according to

$$
\begin{aligned}
& v_{n+1}=v_{n}+\Delta t\left(\frac{D v}{D t}\right)_{n} ; \rho_{n+1}=\rho_{n}+\Delta t\left(\frac{D \rho}{D t}\right)_{n} \\
& \boldsymbol{\sigma}_{n+1}=\boldsymbol{\sigma}_{n}+\Delta t\left(\frac{D \boldsymbol{\sigma}}{D t}\right)_{n} ; r_{n+1}=r_{n}+\Delta t \cdot v_{n}+0 \cdot 5 \Delta t^{2}\left(\frac{D v}{D t}\right)_{n}
\end{aligned}
$$

Time-step control is dependent on the Courant-FriedrichsLewy (CFL) condition, the forcing terms and the viscous diffusion term. Avariable time step is calculated according to Monaghan \& Kos (1999)

$$
\begin{aligned}
\Delta t_{\mathrm{f}} & =\min \left(\sqrt{\frac{h}{\left|f_{a}\right|}}\right), \Delta t_{\mathrm{cv}}=\min _{a}\left(\frac{h}{c_{\mathrm{s}}+\max _{b}\left(h v^{a b} r^{a b} / r_{a b}^{2}\right)}\right), \\
\Delta t & =N_{\mathrm{CFL}} \times \min \left(\Delta t_{\mathrm{f}}, \Delta t_{\mathrm{cv}}\right)
\end{aligned}
$$

Here the CFL number is $0 \cdot 2, \Delta t_{\mathrm{f}}$ is based on the force per unit mass $\left|f_{a}\right|$, and $\Delta t_{\mathrm{cv}}$ combines the Courant and the viscous time-step controls. An automatic time-step adjustment technique is also developed to reduce the occurrence of overestimation of stress when the stress status approaches the plastic yield stage.

\section{MODEL VALIDATION}

Two typical examples are used in this section to verify the validity of the 3D SPH model of large deformation and slope failure. A 2D cohesive soil slope instability test is simulated by a strength reduction technique (Bui et al., 2011), which shows the good applicability of this model to a clay slope. A test involving 3D collapse of a granular slope is reproduced to validate the reliability of this method in simulating the very large deformation of granular materials.

\section{$2 D$ cohesive soil slope instability}

Bui et al. (2011) simulated a 2D cohesive soil slope failure process induced by the strength reduction technique.
The slope is $15 \mathrm{~m}$ high and $45 \mathrm{~m}$ long in total, and the far field boundaries are set to smooth walls, which provide only normal constraints of stress and strain. The length of the inclined part is $22.36 \mathrm{~m}$ and the initial slope angle is $26.57^{\circ}$. The soil parameters of the clay slope are given in Table 1 . A more detailed setting can be found in Bui et al. (2011). Here this work is repeated and a comparison is made with their simulated results. The strength reduction technique is used and the corresponding parameters $c_{\mathrm{r}}$ and $\phi_{\mathrm{r}}$ can be expressed as follows

$$
c_{\mathrm{r}}=\frac{c}{\mathrm{SRF}}, \phi_{\mathrm{r}}=\arctan \left(\frac{\tan \phi}{\mathrm{SRF}}\right)
$$

where $c$ and $\phi$ are the actual shear strength parameters; and $\mathrm{SRF}$ is the shear strength reduction factor. The actual $c$ and $\phi$ are used in the simulation to reach the initial steady state first, then the strength is suddenly reduced to $c_{\mathrm{r}}$ and $\phi_{\mathrm{r}}$ (similar to an earthquake situation; the moment that the strength was reduced is labelled $T=0$ ); finally the simulation evolves to the steady state.

The simulated steady state after slope failure is shown in Fig. 1, the shaded dots represent particles in the simulation and the shading is for the accumulated plastic strain (ADPS), which can represent the slip zone. It can be seen that the final slope profile is highly consistent with Bui's simulated results (black dashed-dotted line), and the form of the slip surface shows good agreement with the Bishop' circle obtained using the limit equilibrium method (black dashed line). This simulation shows the validity of the proposed 3D SPH model for large deformation failure evolution in the $2 \mathrm{D}$ case.

\section{$3 D$ collapse of non-cohesive granular cuboid}

Liu et al. (2013) studied the 3D dynamic collapse process and the internal deformation characteristics of a granular slope in the laboratory. In their experiments, a box $(0.3 \mathrm{~m}$ long, $0.25 \mathrm{~m}$ wide and $0.2 \mathrm{~m}$ high) with a vertical gate on one side of the longitudinal direction is filled with granular materials with a surface elevation of $0 \cdot 144 \mathrm{~m}$. When the gate is lifted vertically and quickly, the granular cuboid begins to collapse and spread. The dynamics in the collapse process is then revealed and the influence of different bases is discussed. The present authors simulated the test conducted on a sand base and rendered the entire collapsing process essentially under the same experimental conditions. To ensure the foundation was covered by more layers of particles and to reduce the influence of boundaries, the base thickness was set to $0.036 \mathrm{~m}$ in the simulation instead of $0.012 \mathrm{~m}$ in the experiment, and the particle spacing of $0.004 \mathrm{~m}$ was chosen to control the calculation consumption. The soil parameters in this simulation are listed in Table 1 as sample 2.

Liu et al. (2013) focused on the final topography of the slope and the spatial and temporal distribution of internal deformation structures. Here the focus is on numerical reproduction of two key experimental data: the final topography obtained from 3D scanner and the internal deformation structures obtained by a shaded line separating different layers. Fig. 2 shows the simulated final shape after the dumping of the $3 \mathrm{D}$ granular slope. A general $3 \mathrm{D}$ view of

Table 1. Soil parameters used in this study

\begin{tabular}{l|c|c|c|c|c|c|c}
\hline & $E: \mathrm{kPa}$ & $v$ & $\rho: \mathrm{kg} / \mathrm{m}^{3}$ & $c: \mathrm{kN} / \mathrm{m}^{2}$ & $\phi: \operatorname{deg}$ & $\psi: \operatorname{deg}$ & SRF \\
\hline Sample 1 & 100 & $0 \cdot 3$ & 2000 & 10 & 20 & 9 & $1 \cdot 40$ \\
Sample 2 & 10 & $0 \cdot 25$ & 1530 & 0 & 33 & 0 & $1 \cdot 00$ \\
Case study & 100 & $0 \cdot 3$ & 1800 & 10 & 20 & 9 & $1 \cdot 40$ \\
\hline
\end{tabular}




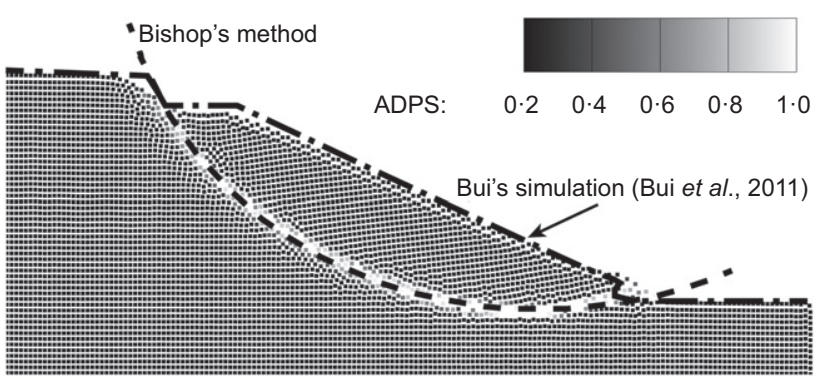

Fig. 1. Simulated steady state of sample 1 after slope failure, as against Bui's simulated results (black dashed-dotted line) and the Bishop' circle from the limit equilibrium method (black dashed line): the shaded dots represent particles under simulation and the shade represents the accumulated plastic strain (ADPS)

the slope shaded according to total displacement is shown in Fig. 2(a), and the quantitative comparison of the elevation contour line can be found in Figs 2(b)-2(d). It can be seen that the numerical results overestimate the elevation of the middle plane a little, whereas they underestimate the width of the slide slightly at the lower parts. Generally speaking, however, the simulated results are satisfactory.

Revealing the internal structure is a distinguishing feature of the work of Liu et al. (2013). They used a coloured line to distinguish different layers and conjectured the internal structures with the deformation of these lines. The current simulation follows the same idea, but only the most basic internal structures are clearly represented (Fig. 3) owing to the limit of spatial resolution. In Fig. 3, the different layers are shaded differently and dashed lines are drawn at the interface of the different layers. A line connecting the inflection point of the dashed lines implies the location and shape of the internal plastic zone. It can be seen that the simulated inflection points of each layers agree well with the observed internal structures (the solid lines), especially the lowest and the second uppermost lines, considering the difficulty of representing the internal structure and limited spatial resolution. These results suggest that the proposed SPH model for slope deformation and failure can effectively simulate the complete process of large deformation and slope failure in the $3 \mathrm{D}$ case.

\section{COMPUTATIONAL CASE STUDIES}

The proposed method was applied to two special 3D slopes with different geometric configurations, including two types of slope: a curving slope surface and turning corners. The detailed geometric configuration is shown in Fig. 4 and the boundary is considered as a rigid wall for all situations. The soil parameters involved are given in Table 1, listed as 'Case study', for both of the two cases.

\section{Case 1: Effect of curvature on 3D slope deformation}

Most natural slopes finally evolve to a certain radian state. According to the 2D plane strain theory, it is not possible to conduct fully the 2D simulation for this type slope with a curving surface. Currently, studies on 3D simulation of slope deformation and failure are rare, and few researchers focus on the effect of geometries on slope deformation. Here, three typical slope deformation and failure processes were simulated, including concave, common and convex surfaces at the same inclination of $45^{\circ}$, as shown in Figs 4(a)-4(c).

Figure 5 shows the final 3D profile for case 1 after deformation. Figs 5(a), 5(b) and 5(c) show the concave slope shaded according to displacement, slope body and cut section of $\mathrm{M}$ and $\mathrm{N}$ planes shaded according to ADPS, respectively; Figs 5(d), 5(e) and 5(f) show the same items for the common slope, whereas Figs 5(g), 5(h) and 5(i) show the items for the convex slope, respectively. The 3D effect on slope deformation is clearly presented, and the whole slip surface eventually evolves to a dish shape. For the three
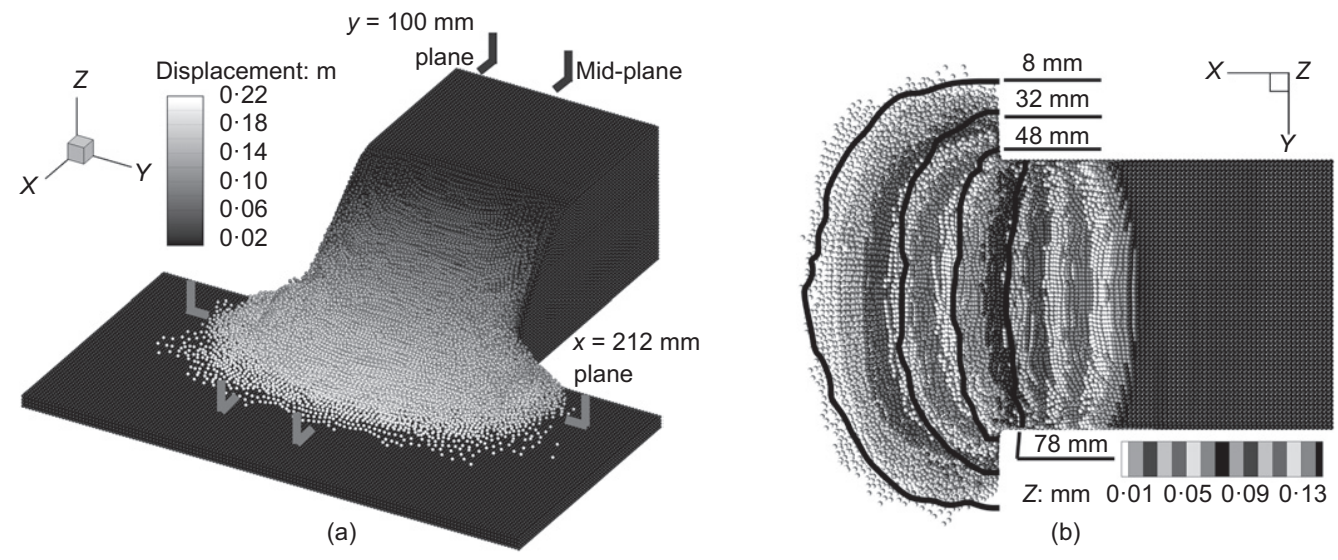

(b)

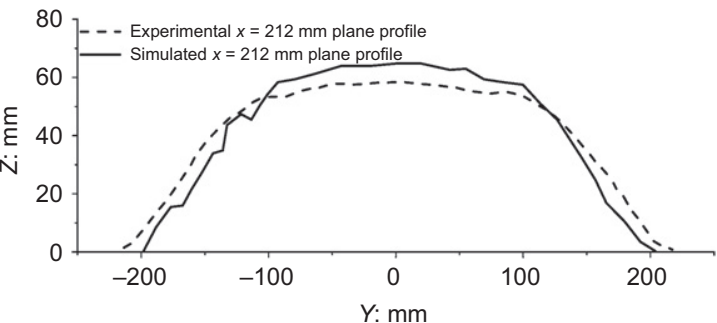

(c)

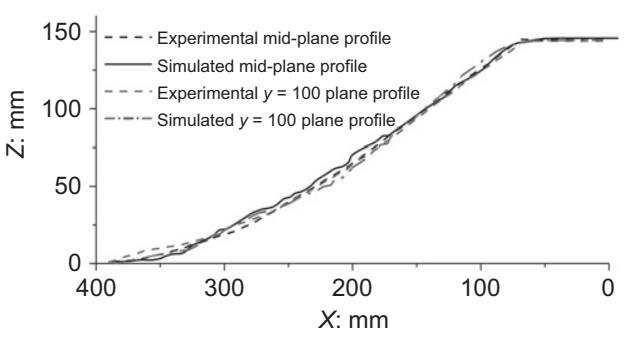

(d)

Fig. 2. The final shape after the collapse of the 3D granular cuboid: (a) 3D view of the slope shaded according to total displacement of each particle; (b) comparison of slip shape between simulated and experimental results from the top view (the simulated result is shaded according to elevation and the black line is the observed contour line in the experiments); (c) quantitative comparison of observed profile and simulated profile at $x=212 \mathrm{~mm}$ plane; (d) quantitative comparison of observed profiles and simulated profiles at middle plane and $y=100 \mathrm{~mm}$ plane 


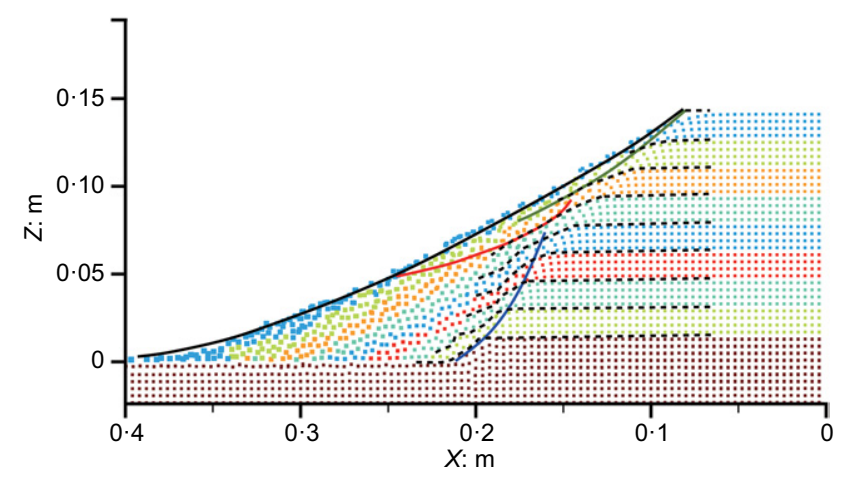

Fig. 3. Comparison of internal deformation within the middle section between numerical (dots) and experimental results (lines). The dots are shaded to represent the layers in the experiments; their size is in proportion to the displacement to highlight the internal slip zones. The dashed lines are drawn to help in separating different layers; the solid lines are internal deformational structures observed by Liu et al. (2013)

typical slopes, including concave, common and convex surfaces, the dish shape of the slip surface becomes successively clearer. Moreover, the convex slope, which is not restrained by the boundaries, cause the largest displacement of the three cases, and the volume of the slide body is significantly larger than the others. The accumulated plastic strain distribution of the slide body can be observed in the cut section of the $\mathrm{M}$ and $\mathrm{N}$ planes. As shown in Figs 5(c) and 5(i), the plastic area of the concave slope is much thinner than that of the convex slope, and the common slope falls in between.

The quantitative comparison of displacement of M1 and $M 2$ in the vertical section $M$ is carried out for the $2 \mathrm{D}$ and $3 \mathrm{D}$ cases, as shown in Fig. 6. If the slope is convex, both the settlement distance at M1 and the translational distance at M2 are greater than for the 2D case, whereas the opposite occurs for the concave slope. If the slope is straight, both the settlement distance at M1 and the translational distance at M2 are less than for the 2D case, just as for the concave case.

These phenomena are a direct consequence of the different 3D stress status for the different slope shapes. Taking the $\mathrm{M}$ plane as an example, the planar stresses in the $M$ plane for the different cases should be more or less similar to each other as their shape in the $\mathrm{M}$ plane is the same. However, the normal stress on the $\mathrm{M}$ plane is very different. For the concave situation, the lateral support of the wall boundaries induces strong lateral stress, which provides extra confining pressure to the soil on the $\mathrm{M}$ plane. Moreover, the concave shape will generate an arch in the elevation plane. The longitudinal direction stress in the $\mathrm{M}$ plane will be redistributed to lateral zones by the arch. These two effects together will reduce the driving force and enhance the constraints, which delay the shear failure of the soil. The convex situation is just the opposite, and the common shape situation falls in between these two. Evidence can be found in Fig. 7 where the data for cross-section $M$ are compared with the $2 \mathrm{D}$ case. The delay of shear failure causes a confined single internal deformation zone for the concave situation, whereas the shear failure occurs in a relatively large zone owing to the lack of constraints of the convex situation. In general, the more the slope bulges, the farther the settlement distance of the slope top and the translational distance of the slope toe migrate (see Figs 7(b)-7(d)).

Case 2: Effect of turning a corner on 3D slope deformation

A slope that turns a corner is often encountered in engineering construction, such as in foundation excavation

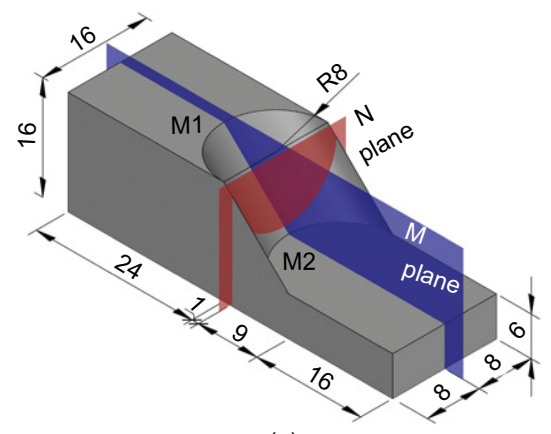

(a)

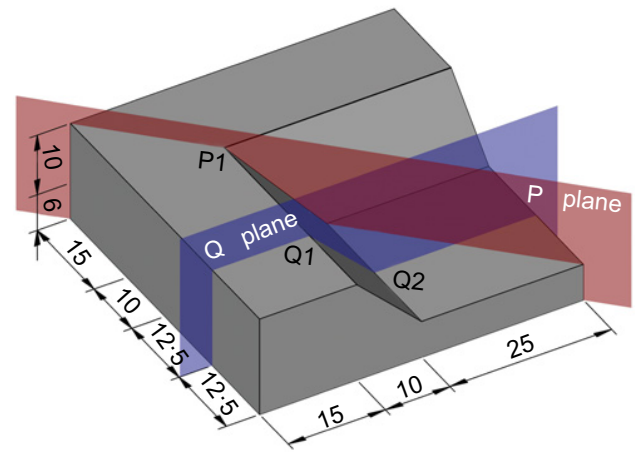

(d)

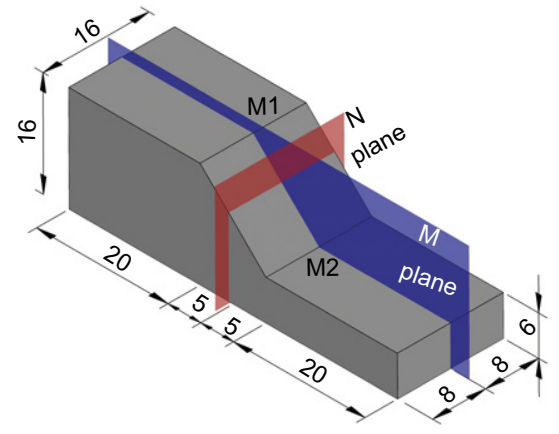

(b)

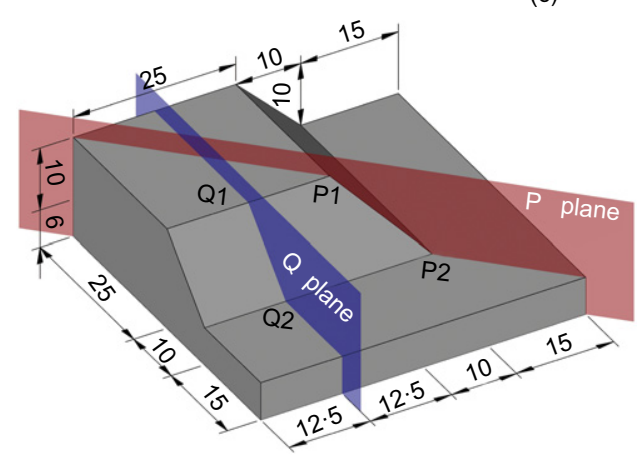

(e)

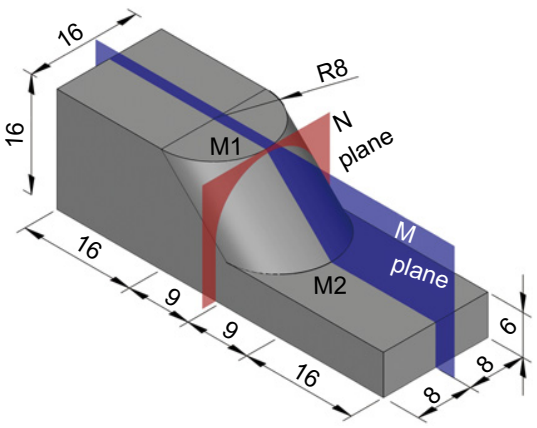

(c) 


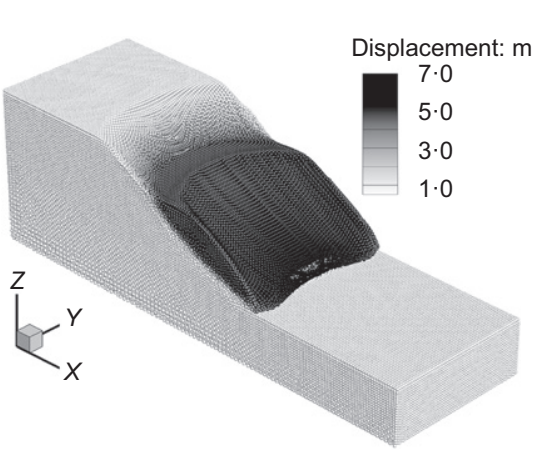

(a)

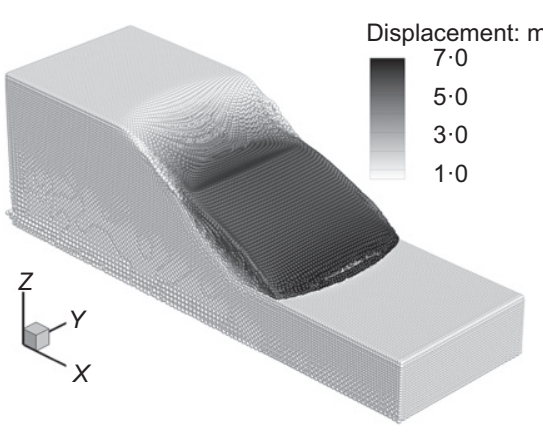

(d)

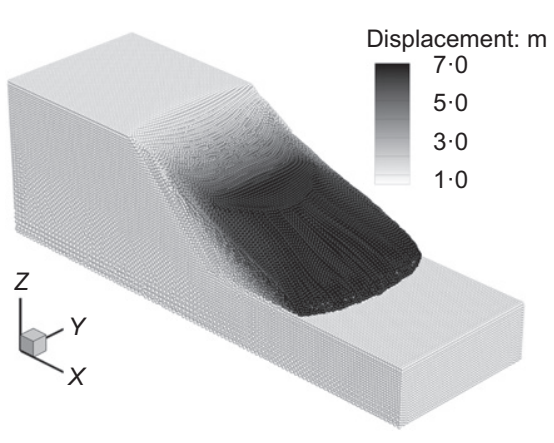

(g)

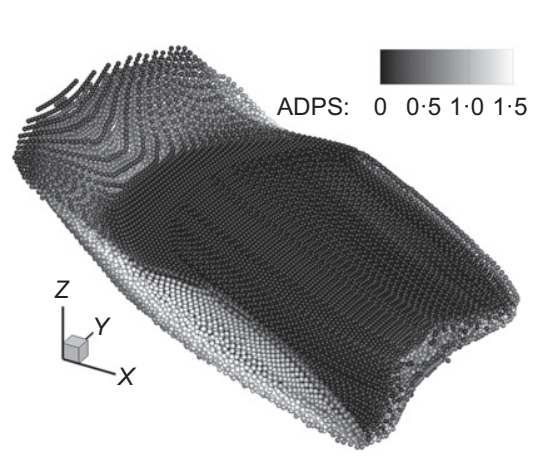

(b)

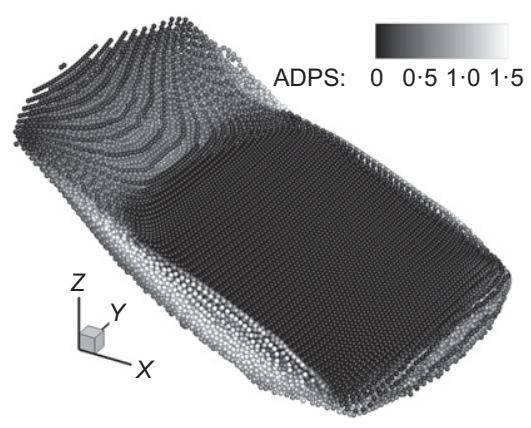

(e)

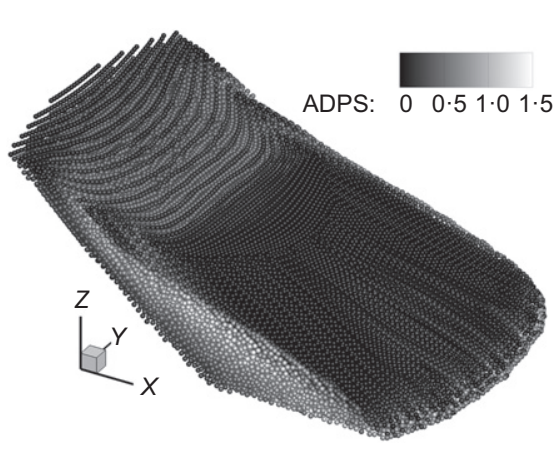

(h)

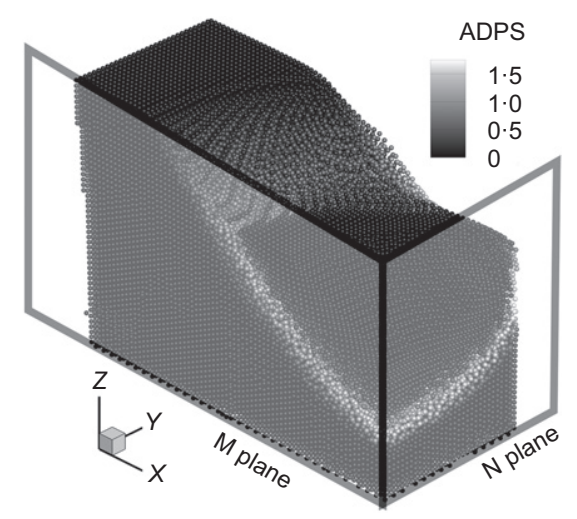

(c)

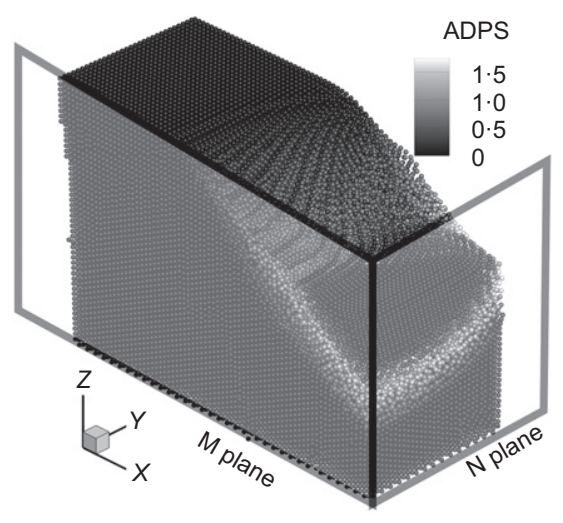

(f)

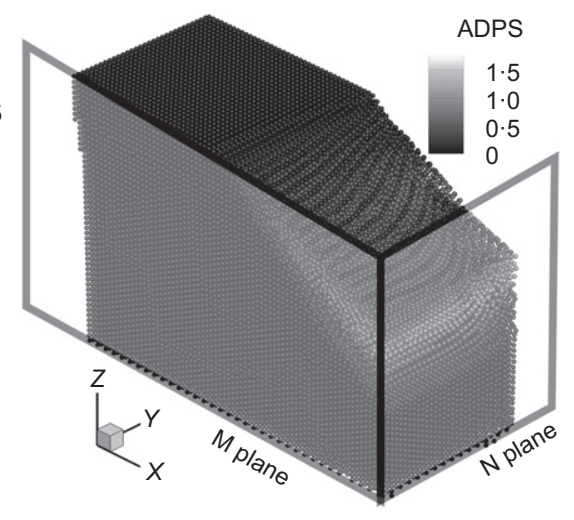

(i)

Fig. 5. Simulated final 3D profile for case 1 after deformation. (a), (b) and (c) show the concave slope shaded by displacement, slide body and cut section of $\mathrm{M}$ and $\mathrm{N}$ plane shaded by ADPS, respectively; (d), (e) and (f) show the equivalent items for a normal slope; (g), (h) and (i) show these items for a convex slope

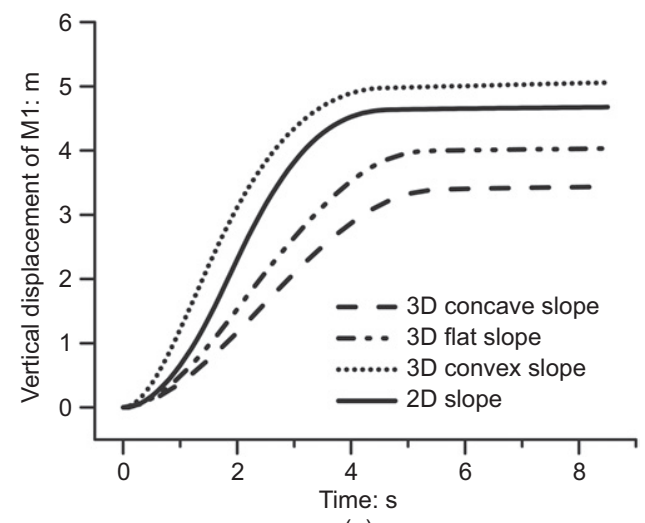

(a)

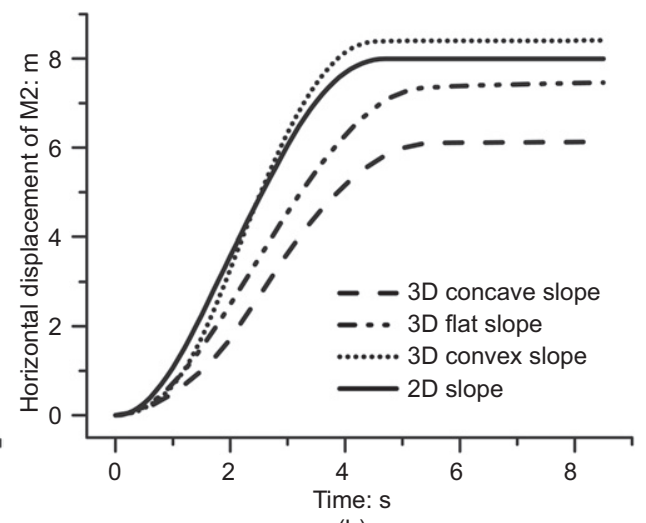

(b)

Fig. 6. Displacement of M1 and M2 in vertical section M: (a) M1 at top of slope; (b) M2 at toe of slope 


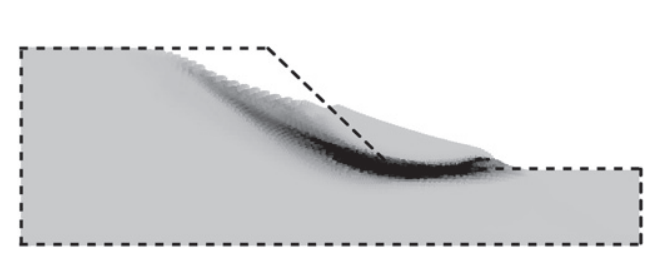

(a)

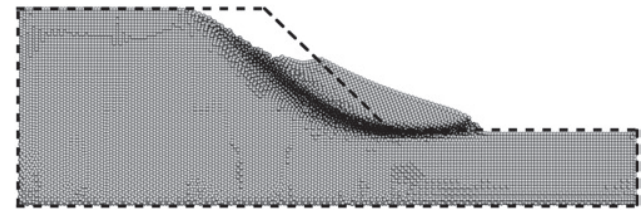

(c)

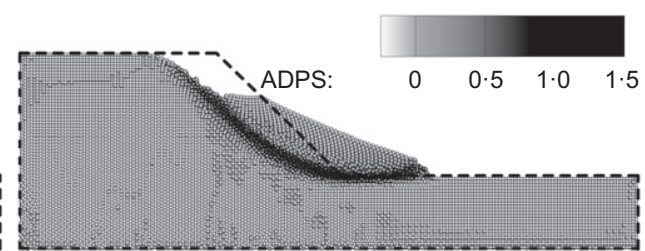

(b)

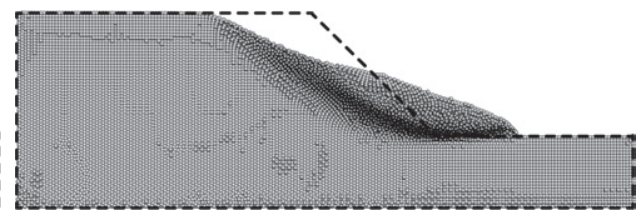

(d)

Fig. 7. Comparison of the cumulative plastic strain between (a) the $2 \mathrm{D}$ condition and the $3 \mathrm{D}$ condition of (b) concave, (c) common and (d) convex slopes in section M. Shaded dots represent the simulation results and the black dashed line represents the initial shape of the slope in two dimensions and the slope section in three dimensions

and embankment slope protection. However, the $2 \mathrm{D}$ simulation could not accurately represent the deformation characteristics for the corner at all. Here, therefore, two typical conditions are considered: the concave and convex sets with a $90^{\circ}$ corner and $45^{\circ}$ side slope. The geometry of the 3D slope model is given in Fig. 4 for: concave (Fig. 4(d)) and convex (Fig. 4(e)) subbase. P1 and P2 are at the top and the toe of the slope in section P; and Q1 and Q2 are at the top and the toe of the slope in section $\mathrm{Q}$.

The final 3D profile after slope deformation is shown in Fig. 8 for the concave (Figs 8(a), 8(b) and 8(c)) and the convex (Figs 8(d), 8(e) and 8(f)) subbases. For each geometric configuration, the overall concave corner slope shaded according to displacement, ADPS and slide body shaded by displacement is shown, respectively. The slip surface of the concave subbase is formed by the hollow junction of two ' $C$ ' surfaces, like a rising wing with a symmetrical shape. For the convex sets, the sliding surface is made up of the raised combination of two ' $S$ ' curves, like the downbeat of a wing with a symmetrical shape. There are two reasons why the displacement at the centre section is smaller than that at the lateral section: first, the slope angle of the slope at the centre is smaller, which make it more stable, and second, the slope at the centre suffers less constraint of the lateral zones.

The quantitative comparison of the vertical displacement of slope toe $\mathrm{P} 1$ and the horizontal displacement of slope toe $\mathrm{P} 2$ in section $\mathrm{P}$ with the 2D case is shown in Fig. 9. This shows that the peak P1 of the concave subbase is almost immobile, and the vertical displacement at P1 of the convex subbase falls in between the 2D case for sections $\mathrm{P}$ and $\mathrm{Q}$. At the slope toe $\mathrm{P} 2$, the horizontal displacement for the concave and convex subbases is small and far below the $2 \mathrm{D}$ case in cross-section $\mathrm{P}$. The 2D case in cross-section $\mathrm{Q}$ is higher than that in section P. Fig. 9 also show a quantitative comparison of the vertical displacement of slope toe Q1 and the horizontal displacement of slope toe Q2 in section $\mathrm{Q}$ with the $2 \mathrm{D}$ case. The vertical displacement at $\mathrm{Q} 1$ of the concave subbase falls between the 2D case for sections $\mathrm{P}$ and $\mathrm{Q}$, as does the horizontal displacement at Q2. For the convex

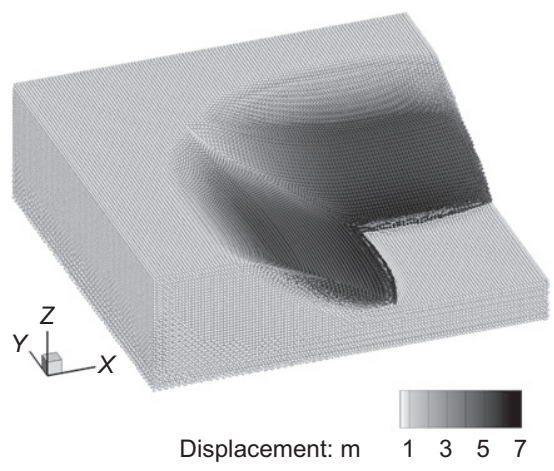

(a)

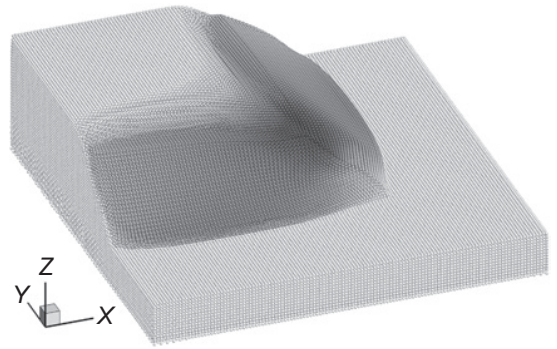

(d)

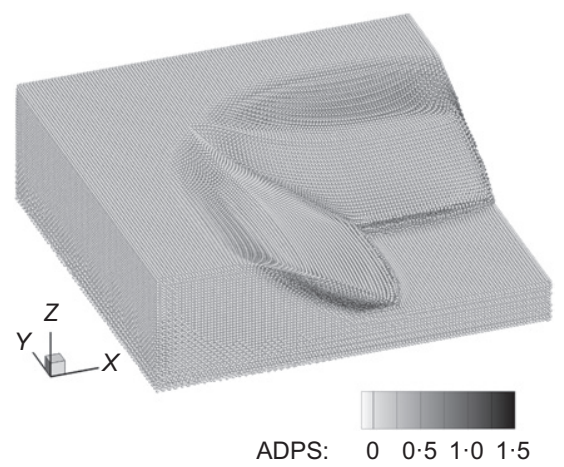

(b)

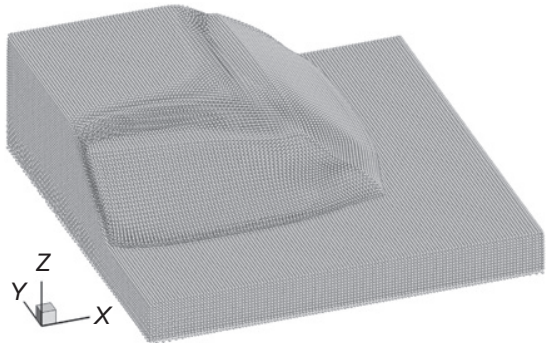

(e)

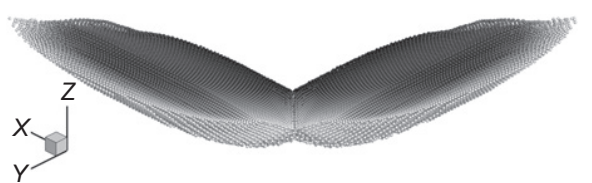

Displacement: $m \quad 1 \quad 3 \quad 5 \quad 7$

(c)

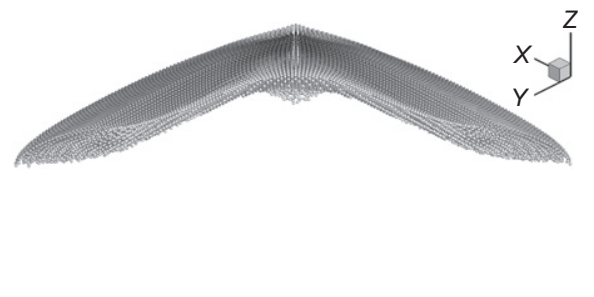

(f)

Fig. 8. Final 3D profile of case 2 after deformation. (a), (b) and (c) show the overall concave corner slope shaded according to displacement, ADPS and slide body, respectively; (d), (e) and (f) show the equivalent items for the convex corner slope 


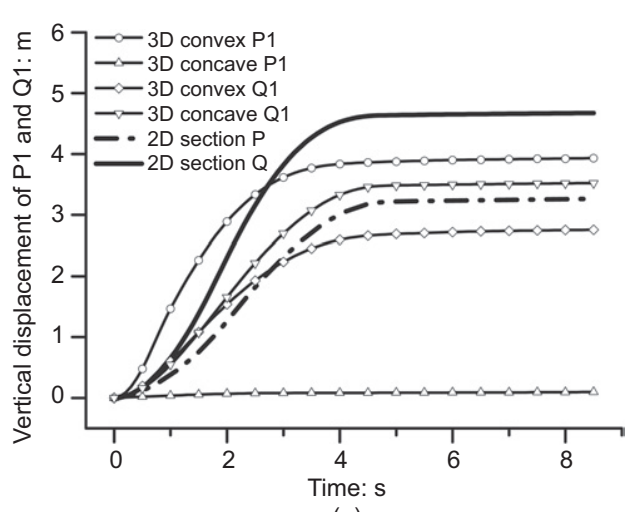

(a)

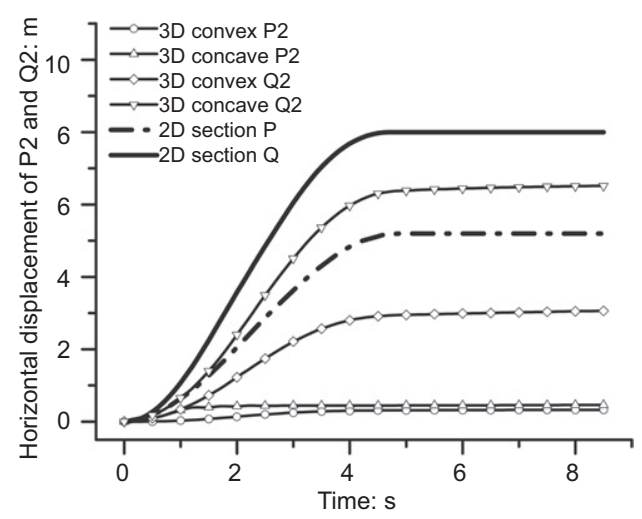

(b)

Fig. 9. Displacement of $P 1 Q 1$ and $P 2$ Q2 in the centre section $P$ and lateral section $Q$ : (a) $P 1$ Q1 at top of slope; (b) P2 Q2 at toe of slope

subbase, the horizontal displacements at Q1 and Q2 are smaller than their corresponding $2 \mathrm{D}$ case in section $\mathrm{P}$. The above analysis shows that the foot of the slope at the corner of the convex and concave subbase is relatively safe, and instability and deformation are more likely to occur at the top corner of the convex subbase and the side slope of the concave subbase.

The reasons why these phenomena occur are similar to case 1: the different 3D stress status for different slope shapes. In this case, the normal stress on the $\mathrm{P}$ plane from each side has a different direction. For the concave subbase case, these two stresses help the slope in the P plane to remain steady, whereas those in the convex case do the opposite. However, the slope angle of the $\mathrm{P}$ plane is relatively smaller than for the Q plane. Thus, the Q plane will always fail first and then help the $\mathrm{P}$ plane to remain steady, even for the convex case. In the convex situation, the failure of the $\mathrm{Q}$ plane causes sinking of the top of the P plane and prevents its further failure; it also causes cracks around the toe of the $\mathrm{P}$ plane, which actually isolate the toe area and reduce the deformation. These mechanisms can be examined by comparing the ADPS data for the cross-sections of $\mathrm{P}$ and $Q$ with the 2D case, as shown in Fig. 10. It is interesting to note that the plastic area of the concave subbase in section $P$ is triangular (where the slide body is mainly composed of the lateral slide materials), whereas it is quadrilateral for the convex subbase.

\section{Discussion}

These two case studies are very typical engineering problems involving soil large deformation in which 3D effects should be considered. The first one involves different normal stress magnitude, whereas the second one involves different lateral stress direction. These differences will lead to different stress status, different shear failure pattern and thus different results. Furthermore, although these two cases are very simple, there is still no simple relation between the $2 \mathrm{D}$ and $3 \mathrm{D}$ results. Thus, to determine how much the $2 \mathrm{D}$ method overestimates or underestimates the results in complex engineering practices remains a challenging task. The method in this paper might be a promising step.

Although the non-Newton fluid-based methods have been proven to be effective in dealing with fluid-like landslides, the method in this paper has its own advantages. As the soil is modelled as elasto-plastic material, which is more general for simulating soil deformation, this method has three advantages when compared with non-Newton fluid-based methods. First, as the elasto-plastic constitutive law is used, this numerical model can simulate not only fluid-like landslides, but also landslides in the steady or quasi-steady state. Second, as all of the nine components of the stress tensor are considered, the stress status would be closer to the real situation, which is essential for the $3 \mathrm{D}$ simulation. For example, the values of $\sigma_{11}, \sigma_{22}, \sigma_{33}$ might be different from each other in the symmetric plane for cases shown in the

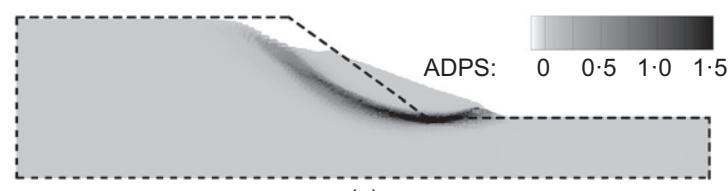

(a)

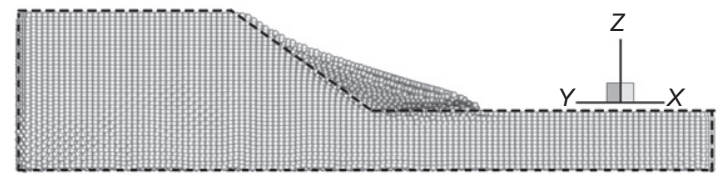

(b)

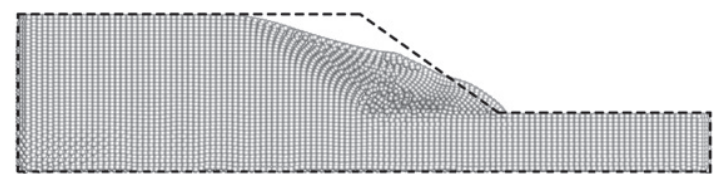

(c)

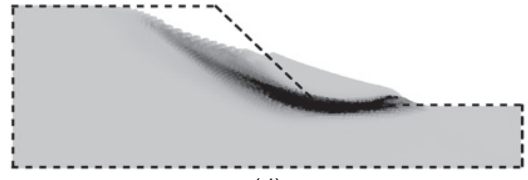

(d)

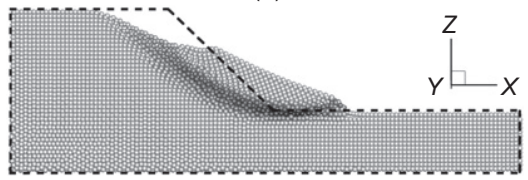

(e)

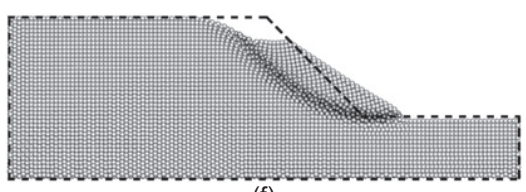

(f)

Fig. 10. Comparison of the cumulative plastic strain between: (a) and (d) 2D condition; (b) and (e) 3D concave slope; (c) and (f) 3D convex slope. (a), (b) and (c) show ADPS in section P; (d), (e) and (f) are for section Q 
section entitled 'Case 1: effect of slope curvature on 3D slope deformation', which could be represented naturally in this study, whereas the fluid-based method would need to use one isotropic pressure to represent this stress status. Third, all parameters in this study could be obtained directly from general soil mechanics experiments, whereas fluid-based methods need antecedent relations, such as the relation between $\tau_{0}$ in the Bingham model and soil parameters $c$ and $\phi$. One of the deficiencies of this method is the huge computational consumption. Solving of the elasto-plastic equations is more complex than for the Navier-Stokes equation, and the small time step to avoid numerical instability exacerbates this problem. The authors are still developing a graphics processing unit accelerated code.

\section{CONCLUDING REMARKS}

This paper develops a SPH-based model for large deformation and failure of a 3D slope. The Drucker-Prager model with non-associated plastic flow rules is implemented into the SPH formulations to describe the whole process of slope failure, including occurrence, development and final stability. Two typical examples of slope instability in two dimensions and in three dimensions are simulated to validate the accuracy and effectiveness of this model. Finally, two case studies on special 3D slope instability with different geometric configurations, including a curving slope surface and a slope that turns corners, are conducted for an exploratory analysis of the final profile, slip surface and distance. Furthermore, qualitative and quantitative comparisons are made with the $2 \mathrm{D}$ cases.

The results show the following.

(a) The simulation of a landslide must consider the 3D effect, as the 3D geometry affects the final profile, slip surface and distance; 2D simulated results are different from $3 \mathrm{D}$ simulation, and the difference is related to the initial slope surface.

(b) For three typical slopes (concave, common and convex), the dish shape of the slip surface becomes progressively and successively more evident; the plastic area of the concave slope is much thinner than that of the convex slope, and the common slope falls in between these two. Comparing with the 2D case, for the convex slope, the settlement distance of the top of the slope and the translational distance of the slope toe are greater than for the 2D case, whereas the opposite occurs for concave and common slopes. These are all related to lateral stress magnitude at the middle plane.

(c) For the two typical subbases (concave and convex), the 3D effect becomes more apparent. The slip surface of the concave subbase is similar to a rising wing, and that of the convex subbase is like a down-beating wing with a symmetrical shape. The toe at the corner of the convex and concave subbases is relatively safe, and instability and deformation at the top corner of the convex subbase and the side slope of the concave subbase are more likely to occur.

These results provide a more accurate and detailed reference for landslide evaluation and foundation ditch design.

\section{ACKNOWLEDGEMENTS}

This work was financially supported by the National Basic Research Program of China (no. 2014 CB04680202) and the National Natural Science Foundation of China (no. 11372326, no. 11432015).

\section{NOTATION}

$c$ cohesion coefficient

$c_{\mathrm{s}}$ speed of sound

E Young's modulus

e deviatoric shear strain rate tensor

$f$ acceleration caused by external force

$f\left(I_{1}, J_{2}\right)$ yield condition

$G$ shear modulus

$g\left(I_{1}, J_{2}\right) \quad$ plastic potential function

$I_{1}$ first invariants of stress tensor

$J_{2}$ second invariants of stress tensor

$K$ elastic bulk modulus

$P \quad$ soil hydrostatic pressure

$\boldsymbol{R}$ artificial stress tensor

$\dot{\boldsymbol{s}}$ deviatoric shear stress rate tensor

$v$ velocity

$W_{i j} \quad$ kernel function

$\alpha, \beta$ Einstein convention in Cartesian components $x, y, z$

$\alpha_{\mathrm{D}}$ normalised coefficient

$\alpha_{\phi}, k_{\mathrm{c}} \quad$ Drucker-Prager's constants

$\Delta t$ time step

$\delta$ Kronecker's delta

$\varepsilon$ constant

$\dot{\varepsilon}$ total strain rate tensor

$\dot{\lambda}$ rate of change of plastic multiplier

$v$ Poisson ratio

$\Pi$ artificial viscosity term

$\rho$ soil density

$\boldsymbol{\sigma}$ total stress tensor

$\phi$ internal friction angle

$\psi$ dilatancy angle

$\dot{\omega}$ spin rate tensor

\section{REFERENCES}

Bui, H. H. \& Fukagawa, R. (2013). An improved SPH method for saturated soils and its application to investigate the mechanisms of embankment failure: case of hydrostatic pore-water pressure. Int. J. Numer. Analyt. Methods Geomech. 37, No. 1, 31-50.

Bui, H. H., Fukagawa, R., Sako, K. \& Ohno, S. (2008). Lagrangian meshfree particles method (SPH) for large deformation and failure flows of geomaterial using elastic-plastic soil constitutive model. Int. J. Numer. Analyt. Methods Geomech. 32, No. 12, 1537-1570.

Bui, H. H., Fukagawa, R., Sako, K. \& Wells, J. C. (2011). Slope stability analysis and discontinuous slope failure simulation by elasto-plastic smoothed particle hydrodynamics (SPH). Géotechnique 61, No. 7, 565-574, http://dx.doi.org/10.1680/ geot.9.P.046.

Cascini, L., Cuomoa, S., Pastorb, M., Sorbinoa, G. \& Piciulloa, L. (2014). SPH run-out modelling of channelised landslides of the flow type. Geomorphology 214, No. 2, 502-513.

Chen, W. \& Qiu, T. (2014). Simulation of earthquake-induced slope deformation using SPH method. Int. J. Numer. Analyt. Methods Geomech. 38, No. 3, 297-330.

Dey, R., Hawlader, B., Phillips, R. \& Soga, K. (2015). Large deformation finite-element modelling of progressive failure leading to spread in sensitive clay slopes. Géotechnique $\mathbf{6 5}$, No. 8, 657-668, http://dx.doi.org/10.1680//geot.14.P.193.

Griffiths, D. V. \& Marquez, R. M. (2007). Three-dimensional slope stability analysis by elasto-plastic finite elements. Géotechnique 57, No. 6, 537-546, http://dx.doi.org/10.1680/geot.2007.57.6. 537.

Hu, M., Liu, M. B., Xie, M. W. \& Liu, G. R. (2015). Three-dimensional run-out analysis and prediction of flow-like landslides using smoothed particle hydrodynamics. Environ. Earth Sci. 73, No. 4, 1629-1640.

Huang, Y. Zhang, W. J., Xu, Q., Xie, P. \& Hao, L. (2012). Run-out analysis of flow-like landslides triggered by the Ms 8.0 2008 Wenchuan earthquake using smoothed particle hydrodynamics. Landslides 9, No. 2, 275-283.

Liu, G. R. \& Liu, M. B. (2004). Smoothed particle hydrodynamics: a meshfree particle method, 1st edn. Singapore: World Scientific.

Liu, Z., Koyi, H. A., Swantesson, J. O. H., Nilfouroushan, F. \& Reshetyuk, Y. (2013). Kinematics and 3-D internal deformation 
of granular slopes: analogue models and natural landslides. J. Struct. Geol. 53, No. 4, 27-42.

Monaghan, J. J. \& Kos, A. (1999). Solitary waves on a Cretan beach. J. Waterway, Port, Coastal Ocean Engng 125, No. 3, $145-154$.

Nian, T. K., Huang, R. Q., Wan, S. S. \& Chen, G. Q. (2012). Three-dimensional strength-reduction finite element analysis of slopes: geometric effects. Can. Geotech. J. 49, No. 5, 574-588.

Pastor, M., Haddad, B., Sorbino, G., Cuomo, S. \& Drempetic, V. (2009). A depth-integrated, coupled SPH model for flow-like landslides and related phenomena. Int. J. Numer. Analyt. Methods Geomech. 33, No. 2, 143-172.

Tapias, M., Alonso, E. E. \& Gili, J. (2015). A particle model for rockfill behaviour. Géotechnique 65, No. 12, 975-994, http:// dx.doi.org/10.1680/jgeot.14.P.170.

Yang, X. F. \& Liu, M. B. (2012). Improvement on stress instability in smoothed particle hydrodynamics. Acta Physica Sinica 61, No. 22, 224701 (in Chinese).

Zhang, Y. B., Chen, G. Q., Zheng, L., Li, Y. G. \& Zhuang, X. Y. (2013). Effects of geometries on three-dimensional slope stability. Can. Geotech. J. 50, No. 3, 233-249. 